\title{
Abordaje del tema 'Internacionalización de la Educación Contable' \\ en espacios académicos brasileños
}

\section{Xiomara Esther Vazquez Carrazana, Gilberto José Miranda, Marli Auxiliadora da Silva*}

\author{
Programa de Pós Graduação em Ciências Contábeis (Pós Doutorado) \\ Universidade Federal de Uberlândia \\ Programa de Pós Graduação em Ciências Contábeis \\ Universidade Federal de Uberlândia \\ Faculdade de Administração, Ciências Contábeis, Engenharia de Produção e Serviço Social \\ Universidade Federal de Uberlândia
}

Este estudio tiene como objetivo analizar cómo ha sido discutido el tema de las International Education Standards (IES) en el espacio académico brasileńo. Para ello, se utilizó una metodología documental para revisar los artículos científicos publicados en la base de datos Scientific Periodicals Electronic Library (SPELL), el contenido de la disciplina didáctico-pedagógica impartida en los programas de posgrado y la producción derivada de la culminación de estudios de estos cursos. Esta revisión fue realizada para el período 2010-2017. Los resultados obtenidos permitieron considerar que, en los espacios académicos brasileńos, las IES no son abordadas con la dimensión y profundidad requeridas para la comprensión de sus limitaciones y del arsenal epistemológico necesario para su puesta en práctica. Los estudios desarrollados en Brasil no contemplan las problemáticas generadas en torno a las IES. De esta manera, este artículo supone una alerta sobre el peligro que representa asumir las normativas publicadas sin antes ser sometidas a debate y reflexión crítica para la formación de profesionales contables. Este estudio debe ser superado por otros que evidencien las limitaciones implícitas en cada normativa para liderar el cambio necesario en la educación contable.

Palabras claves: Normativas Internacionales de Educación Contable, educación contable, espacios académicos brasileños

\section{Approach to the topic 'Internationalization of Accounting Education' in Brazilian Academic Spaces}

This study aims to analyze how the topic of International Education Standards (IES) has been discussed in the Brazilian academic space. A documentary methodology was used to review the scientific articles published in the Scientific Periodicals Electronic Library (SPELL) database, the content of the didactic-pedagogical discipline taught in post-graduation programs and the production derived from the culmination of studies of these courses; This review was carried out for the period 2010-2017. The results obtained allowed us to consider that, in the Brazilian academic spaces, the IES are not approached with the dimension and depth required to understand their limitations and the epistemological arsenal necessary for their implementation. The studies carried out in Brazil do not contemplate the problems generated around the IES; This article constitutes an alert on the danger that it represents, for the training of accounting professionals, to assume the published regulations without being subjected to debate and critical reflection. This study must be overcome by others that demonstrate the limitations implied in each regulation to lead the necessary change in accounting education.

Keywords: International Accounting Education Regulations, Accounting Education, Brazilian Academic Spaces

* Agradecemos a las instituciones CAPES y CNPq por el apoyo para la obtención de los resultados expuestos en este artículo. 


\section{Abordagem do Tema 'Internacionalizaçáo da Educação Contábil' nos Espaços Académicos Brasileiros}

Este estudo tem como objetivo analisar como o tema das International Education Standards (IES) tem sido discutido no espaço acadêmico brasileiro. Utilizou-se metodologia documental para revisar os artigos científicos publicados na base de dados da Biblioteca Eletrônica de Periódicos Científicos (SPELL), o conteúdo da disciplina didático-pedagógica ministrada nos programas de pós-graduação e a produção derivada da culminação de estudos de esses cursos; esta revisão foi realizada para o período 2010-2017. Os resultados obtidos permitiram considerar que, nos espaços acadêmicos brasileiros, as IES não são abordadas com a dimensão e profundidade necessárias para entender suas limitaçôes e o arsenal epistemológico necessário à sua implementação. Os estudos realizados no Brasil não contemplam os problemas gerados em torno das IES. Este artigo constitui um alerta sobre o perigo que representa, para a formação de profissionais de contabilidade, assumir as normativas publicados sem ser submetido a debate e reflexão crítica. Este estudo deve ser superado por outros que demonstrem as limitaçóes implícitas em cada normativa para liderar a mudança necessária no ensino contábil.

Palavras-chave: Normativas Internacionais de Educação Contábil, Educação Contábil, Espaços Acadêmicos Brasileiros

\section{Introducción}

Los académicos e investigadores se han preocupado por el estudio de acciones que modifican la oferta educativa actual, pues consideran que es una exigencia derivada de los continuos y trascendentes cambios en la esfera económica y social (Argueta, 2009). En el caso de los cursos de pregrado y posgrado de la profesión contable, estas acciones cobraron fuerza cuando el International Federation of Accountants (IFAC) publicó la International Education Standards (en adelante, IES). En 2008, se publicaron ocho IES con el objetivo de uniformar el contenido programático; las habilidades, los valores, la ética y las actitudes que deben regir la formación contable; y las competencias que deben ser evaluadas en el profesional de esta rama (Castillo, Córdova \& Villarreal, 2014).

Dichas normativas se consideraron importantes, porque se acercan a las metas de la educación contable (Quijano, 2002). Además, establecen los requerimientos mínimos a través de la uniformidad de un conjunto de variables del proceso formativo (Gómez, 2007; Martínez, 2010; Gómez \& Martínez, 2014), y brindan al IFAC puntos de referencias comunes que pueden minimizar las diferencias en la formación de los contadores para su actuación en un contexto de globalización y expansión de los mercados (Da Silva, 2012; Reyes \& Chaparro, 2013; Sugahara \& Wilson, 2013; Helliar, 2013; Watty, Sugahara, Abayadeera, Perera \& Mckay, 2014; Gómez \& Martínez, 2014; Crawford, Helliar, Monk \& Veneziani, 2014; Jacomossi \& Biavatti, 2017). No obstante, la aparición de criterios sobre las insuficiencias de tales normativas para resolver los problemas de la educación contable no se hizo esperar.

Se considera que las IES son un referente incompleto y poseen un alcance restringido para el proceso de enseñanza-aprendizaje de la contabilidad (Gómez, 2007; López, 2013; León, 2014; Zapata \& Sarmiento, 2016), ya que no definen acciones cognitivas para asimilar, interpretar e interactuar en contextos socioeconómicos distintos a los de la gran empresa (Gómez, 2007). Además, las IES manifiestan una orientación excesiva hacia el mercado. En ese sentido, relega la formación integral a un segundo plano (López, 
2013), pues su excesiva concentración en aspectos instrumentales ha subestimado la formación sociohumanística y el pensamiento crítico. Así, evidencian falencias de un panorama académico que no contempla las relaciones sociales subyacentes en el saber contable (Quirós, 2006; Quinche, 2007; Gómez \& Ospina, 2009; Rueda, 2010a; Marulanda, Piedrahita \& Quintero, 2011; Montoya \& Farías, 2013; León, 2014; Rojas \& Giraldo, 2015; Muñoz, Ruiz \& Sarmiento, 2015; Zapata \& Sarmiento, 2016; Ramírez, 2017). La aceptación de las IES, sin ser sometidas a profundas discusiones conceptuales, metodológicas y axiológicas, perpetuará la prioridad por el aprendizaje rígido presente en los programas de formación de los contadores de pregrado y posgrado. De esa manera, se limita el desarrollo del pensamiento de estos profesionales para la comprensión y transformación de los problemas presentes en la práctica actual (Rueda, 2010b; Rueda, Pinzón \& Patiño, 2013; Zapata \& Sarmiento, 2016).

Las insuficiencias intrínsecas en las IES, reveladas con mayor énfasis por los autores latinos de idioma español, ponen sobre el tapete los siguientes aspectos: 1) las IES son un punto de partida importante para el cambio requerido en la educación contable; 2) el IFAC orienta que deben ser consideradas como modelo durante el proceso de calificación y desarrollo permanente de los contadores, aunque no tengan un carácter prescriptivo; 3) no deben ser asumidas sin antes comprender sus limitaciones para la formación integral; y 4) será imprescindible discernir su contenido epistemológico. Estas reflexiones motivaron la siguiente pregunta: ¿cuál es la repercusión de las IES en el pensamiento contable en Brasil? En ese sentido, el objetivo del presente estudio es analizar cómo ha sido discutido el tema de las IES en el espacio académico brasileño.
A partir de la revisión previa de la literatura sobre el tema, se ha evidenciado que los autores brasileños revelan temor ante el peligro de que el proceso formativo de contadores en el país esté descuidando las tendencias internacionales en la educación contable (Czesnat, Cunha \& Domingues, 2009; Miranda, 2010; Erfurth \& Carvalho, 2013; Jacomossi \& Biavatti, 2017). Aun así, se observa un discurso pasivo en torno al tema, pues no se alerta sobre el peligro que implicaría asumir las normativas como sentencias finales sin una visión crítica, reflexiva y propositiva para responder a los actuales problemas del proceso formativo contable.

\section{Generalidades}

Generalmente, la contabilidad es concebida como ciencia social. Cabe señalar que una ciencia no es social por derecho propio. Una ciencia social debe ser capaz de desarrollar formulaciones conceptuales sobre su entorno (Larrinaga, 1999). En ese sentido, surge la preocupación sobre las limitaciones del proceso formativo contable para el análisis crítico de contextos sociales (Quirós, 2006; Gómez, 2011; Gracea, 2014; Rocancio, Mira \& Muñoz, 2016), ya que los programas académicos contables sufren con la saturación de contenidos descriptivos y poco reflexivos (Martínez, 2007).

Por regla general, el currículo de contabilidad se estructura desde una concepción de regulación contable y empírica con escasas iniciativas frentes a los saberes y al conocimiento. Esto ha imposibilitado el desarrollo de competencias para la identificación de las problemáticas de la contabilidad frente a su entorno social. Bajo este esquema, la actuación profesional manifiesta rigidez ante la comprensión de las diversas dimensiones que se relacionan con el mundo empresarial (Gracia, 2000). La visión empírica de la 
contabilidad descarta otras aproximaciones (Rueda, 2007): al refugiarse en lo instrumental, proyecta una visión mecanicista del saber; esto limita el debate para consolidar una conducta disciplinaria que reconozca las circunstancias contextuales (León, 2014). Tal desequilibrio, a favor de lo instrumental, imposibilita el diálogo entre los diversos campos del saber (Romero, 2014; León, 2014; Major, 2017). Entonces, su discurso es parcelado (García, 2014), y no vigila el espíritu reflexivo, autónomo y crítico necesario en la profesión contable (Martínez, 2002; Zapata \& Sarmiento, 2016).

El problema que emerge de los procesos formativos del profesional contable ha sido considerado consecuencia del pragmatismo excesivo del currículo que lo desvincula de su función legítimamente formativa (Selter, 2001; Galagovsky \& Adúriz, 2001; Martínez, 2008; León, 2009; Cuenú, 2010; Sánchez, 2011; García, 2014). Esto contribuye con la crisis de los argumentos teóricos que sustentan la práctica contable (Martínez, 2007; Rueda \& Pinzón, 2009; Rueda, 2010a; León, 2014; Rojas \& Giraldo, 2015). La argumentación reflexiva es una de las competencias que posibilita lidiar con opiniones controversiales, lo que la convierte en una de las habilidades más importantes para la actuación de los profesionales en la práctica social (Larraín, Freire, Moretti, Requena \& Sabat, 2015; Carrillo \& Jurado, 2017). Sin embargo, algunos autores consideran que la educación contable no está problematizada filosóficamente, lo cual no permite el desarrollo significativo de evoluciones conceptuales (Rojas, 2002, Galagovsky \& Adúriz, 2001; Nersessian \& Oliva, 2007). De hecho, son escasos los contenidos dedicados a la construcción teórica (Giraldo, 2006; Rojas, 2007; Gómez, 2009; Gómez \& Martínez, 2014), pues se destaca la formación técnica e instrumental (García, 2014).
La crisis de los argumentos teóricos no ha permitido al contador pensar para establecer relaciones significativas entre las posturas de la ciencia contable y otras complementarias (Muñoz et al., 2015). Al observar el desarrollo de la disciplina contable en la universidad, se manifiesta que las diferentes opiniones en la práctica pedagógica coinciden en que el proceso formativo de contadores debe avanzar hacia el abordaje epistemológico y la formación humanística (Martínez, 2007; García, 2014; Rojas \& Giraldo, 2015; Ramírez, 2017).

La formación de competencias es determinante en la formación de los futuros profesionales (Larraín et al., 2015; Carrillo \& Jurado, 2017). Las insuficiencias de las IES hacia esa dirección han marcado el debate, y han evidenciado la necesidad de desentrañar y someter a juicio crítico sus fundamentos y los obstáculos para que las IES sean aceptadas y compartidas como mejores prácticas (Gómez, 2007; Rueda, 2010a; López, 2013; León, 2014; Castillo et al., 2014). Se suponía que una nueva normativa para la educación contable lideraría cambios importantes en el proceso formativo en esta área. Sin embargo, existe un sentimiento de incertidumbres en cuanto a su transcendencia y potencialidades para impulsar el cambio. De hecho, la revisión previa de algunos documentos deja indicios de que esta normativa presenta algunas fisuras conceptuales que no le permiten cumplir el cometido mencionado.

\section{Elementos del debate sobre la Normativa Internacional de Educación Contable}

La visión curricular de las IES excluye la formación humanística como elemento insoslayable de toda práctica académica (Quijano, 2002; Quirós, 2006; García, 2014; Muñoz et al., 2015; Martínez, 2010). De esta manera, ha disminuido la concepción del 
rigor científico en la profesión contable, pues se privilegia un sistema educativo orientado a perfiles ocupacionales que propende a la formación con estrecho marco crítico y reflexivo (Martínez, 2007; Rojas, 2008; García, 2014; Rojas \& Giraldo, 2015; Villarreal, Córdoba \& Castillo, 2016), y no estimula la capacidad de los estudiantes para organizar sustentos teóricos que le permitan enfrentar los problemas del entorno económico actual (León, 2014). El énfasis de la IES reside en un contenido que sobrevalora los componentes técnico-instrumentales, por lo que perpetúa un proceso formativo que asiste al contador solo con competencias básicas. En ese sentido, dichas normativas obstaculizan la movilidad hacia el interior del ejercicio interdisciplinario (Martínez, 2007; León 2014), y provocan que la educación contable continúe priorizando los aspectos técnicos e intereses comerciales estrechos al desligarla de la posibilidad de interpretación hermenéutica propia de las ciencias sociales (Rueda, 2010a; Rueda 2010b; Rueda, 2012).

Estos aspectos han llevado a que, desde el pensamiento contable, se reflexione en torno a la necesidad de estructurar las prácticas pedagógicas de contabilidad filosóficamente para promover la formación integral. Dicha formación no será adquirida solo a través de los cursos específicos propuesto por el IFAC, pues el crecimiento profesional demandará de otras argumentaciones e interacciones (García, 2014; Rojas \& Giraldo, 2015; Cassiani \& Zabaleta, 2016; Villarreal et al. 2016). De esa forma, las IES tendrán que ser sometidas a reflexión pedagógica, ya que no dan respuesta a la formación de un profesional que pueda modificar su entorno de manera razonable. Hay que considerar que no es posible aplicar las Normas Internacionales de Educación Contable si no se discute su soporte epistemológico para que sean pertinentes para el perfeccionamiento del proceso formativo.

Las competencias conforman uno de los temas más polémicos dentro del contenido de las IES. Han sido definidas como habilidades para desempeñar las tareas y los roles esperados de un contador profesional. Tal sentido dista de la concepción de las competencias como categorías conceptuales o integradoras mediante las cuales se podrá comprender, interpretar y transformar los diferentes contextos (López, 2013). La situación debe ser rectificada para avanzar en la reflexión educativa junto al proceso de convergencia de las nuevas dinámicas del ejercicio profesional contable (Izquierdo \& Adúriz, 2003; Tamayo \& Orrego, 2005; Castillo et al., 2014). Una parte considerable de la discusión actual se origina a partir de la definición de "competencia" por la normativa como las posibilidades demostradas para ejecutar trabajos (López, 2013; Villarreal et al., 2016). Considerando este sentido de competencia, el profesional contable difícilmente estaría capacitado para apropiarse con autonomía de procesos de reflexión crítica. En el contenido de las normativas, no están referidas las competencias complejas en función de un pensamiento crítico derivado de la reflexión y la comprensión argumentativa (López, 2013).

Por otro lado, la normativa aborda las habilidades como si fueran independientes de las competencias. Si bien las definiciones de competencias son diversas, la acepción más frecuente es que, de una u otra manera, el ámbito de la habilidad es parte de la competencia; es decir, la competencia es un aprendizaje complejo que integra conocimientos, habilidades, aptitudes, valores y actitudes (González, 2006; Rocancio et al., 2016).

El problema fundamental de la noción de «competencia» de la IES radica en soslayar la formación de competencias cognitivas. Esto sucede porque se ha colocado el trabajo como eje central del proceso formativo; es decir, se reproduce la visión técnica de la 
contabilidad en detrimento de su carácter científico, lo que implica obviar que el proceso formativo de esta rama debe enfocarse en la explicación, compresión e interpretación de las relaciones de causa y efecto de los aconteceres económicos y financieros (Cortés, 2006; Gómez, 2007; González, 2008; Castillo et al., 2014; Sosa, 2015). Perder de vista esta percepción entraña la minusvaloración de los saberes necesarios para que el contador pueda interactuar, explicar y transformar la práctica económico-social (Castillo et al., 2014).

En su intento por que las competencias sean la base del proceso formativo, el IFAC prescribió la evaluación de las competencias. No obstante, las competencias que deben ser formadas no son definidas. Tampoco se explica cómo serán medidas y evaluadas; ni en qué grado se deben desarrollar las mismas, pues algunas competencias solo son incrementadas a lo largo de muchos años. Por otro lado, el requisito de competencias específicas para auditores constituye un problema no resuelto dentro de las normativas, ya que no se abordan las diferencias de las competencias para unos y otros (Needles, 2008).

La ambigüedad en la conceptualización de las competencias condicionó el diseño de contenidos cargados de componentes técnicos, tecnológicos y financieros con énfasis en la necesidad de los negocios y con una fuerte dosis de aspectos estrictamente contables. De esta manera, impera un enfoque multidisciplinario en lugar de interdisciplinario (Gómez, 2009; Gómez \& Ospina, 2009; Rueda, 2012; Castillo et al., 2014; Celerino, 2015; Quintero \& Ramírez, 2016; Ramírez, 2017). Este enfoque ha dejado de lado la condición humanista y social de la contabilidad; por el contrario, enfatiza únicamente la perspectiva funcional e instrumental, y evidencia serias debilidades en el discurrir crítico interpretativo y la necesaria reflexión conceptual (Martínez, 2007; Villarreal et al., 2016). Solo se podrá retomar la reflexión filosófica en la contabilidad si se vincula con otras disciplinas de naturaleza humanista y social (Rojas, 2006; Quinche, 2007; Rueda, 2012; Rueda et al., 2013; Castillo et al., 2014).

Otra de las contradicciones de las IES es que pretenden funcionar como un estándar. En ese sentido, no explicitan la necesidad de que el proceso formativo de los contadores refleje sus propios entornos, y objetivos nacionales y culturales relevantes. De alguna manera, esto puede ser interpretado como una forma de autoritarismo que desconsidera que las dimensiones culturales de cada país afectan significativamente las preferencias de estilo de aprendizaje de los estudiantes (Sugahara \& Boland, 2010; Sugahara, Urasaki, Wei \& Boland, 2010). Las IES no plantean la identidad como imperativo de la calidad educativa (Cardona, 2000), y emplean un único enfoque que no involucra las particularidades y la multiplicidad de usuarios de la información contable (Dellaportas, Leung, Cooper \& Jackling, 2006; Mejías et al., 2008). De esta manera, evidencian desconocer la variada gama de necesidades y de entornos existentes (Mejías, Montes \& Montilla, 2008)

La concepción del proceso de formación de las IES pierde de vista las ópticas más amplias de formación de sujetos sociales integrales. De esta manera, se desconfigura el papel de la educación como el medio por excelencia para consolidar la cultura, y para lograr la adaptación, interacción y transformación sostenible del entorno (Gómez, 2006; Gómez, 2007; Cuenú, 2010; Zapata \& Sarmiento, 2016). La carencia del enfoque socio-formativo implica la negación de una formación humana integral en el marco de relaciones dinámicas con la sociedad, la cultura y el medio ambiente, a pesar de que el reto de la educación contable debería irradiar diferentes contenidos (habilidades, conocimientos y valores) hacia todos los espacios formativos. 
El análisis evidenciado por los diferentes autores deja espacio para dudar sobre las posibilidades de que las IES sean un referente completo para movilizar a los actores del proceso hacia el empleo de las herramientas cognitivo-interpretativas que incentivan las virtudes críticas y creadoras de los profesionales. Por ello, las construcciones epistemológicas necesarias para las ciencias contables se han visto amenazadas.

\section{Metodología}

El estudio realizado se sustenta en una investigación de tipo documental. La investigación se estructuró en las siguientes etapas: (a) revisión de artículos publicados sobre el tema y análisis del contenido de los mismos; (b) revisión del contenido de la asignatura Metodología de la Enseñanza en posgrado; y (c) producción derivada de la culminación de estudio de los estudiantes de posgrado. Se han considerado los siguientes espacios como los principales escenarios académicos: 1) artículos científicos publicados y 2) programas de posgrado. El período de estudio es 2010-2017. Considerando que el IFAC publicó las IES en 2008, se sugiere la existencia de un período de madurez en el análisis y discusión de estas normativas a partir del ańo 2010.

La primera etapa del estudio consistió en recolectar los artículos científicos publicados en la base de dato Scientific Periodicals Electronic Library (SPELL) sobre la temática del estudio. Este proceso se materializó a través de la búsqueda utilizando términos relacionados al tema de investigación: Normas Internacionales de Educación Contable, Internacionalización de Educación Contable, IFAC, IES. En la segunda etapa, se revisaron las páginas web de los cursos de posgrado en Ciencias Contables evaluados por Coordinación de Perfeccionamiento de Personas de Nivel Superior (CAPES) en Brasil. En total, fueron 31 cursos. En esta fase, se revisó el contenido de la disciplina Metodología de la Enseñanza para observar si aborda el contenido de las IES. También se revisaron las tesis y disertaciones defendidas con el objetivo de comprobar el tratamiento del tema como parte de las investigaciones de los programas.

\section{Resultados}

La información sobre los artículos recolectados en la plataforma SPELL se presenta a continuación en la tabla 1. Se han distribuido a partir del Qualis de la revista en la que fueron publicados.

En el período estudiado, se publicaron diecisiete artículos sobre el tema de investigación. Dada la importancia del tema, se considera que la cifra de publicación es pobre. Cabe resaltar que más de la mitad de los artículos fueron publicados en revistas de Qualis inferior a B2, lo que puede ser un indicio de falta de reconocimiento de la investigación en educación

Tabla 1. Distribución de artícullos por Qualis de la revista donde fueron publicados

\begin{tabular}{|l|c|c|c|c|c|c|c|c|c|}
\hline Ranking de las revistas & 2010 & 2011 & 2012 & 2013 & 2014 & 2015 & 2016 & 2017 & Total \\
\hline Qualis A2 & - & 2 & - & 2 & - & - & 1 & - & 5 \\
\hline Qualis B1 & 1 & - & - & - & - & - & - & 1 & 2 \\
\hline Qualis B2 & - & - & - & - & - & 1 & - & - & 1 \\
\hline Outros qualis & 1 & - & 2 & - & 1 & - & 1 & 4 & 9 \\
\hline Total & 2 & 2 & 2 & 2 & 1 & 1 & 2 & 5 & 17 \\
\hline
\end{tabular}

Fuente: Elaboración propia a partir de datos de plataforma SPELL. 
contable. Las valoraciones del proceso de internacionalización de la educación contable fueron realizadas a partir de estudios bibliométricos, y comparaciones de currículos entre universidades o países con un enfoque de generalización y ausencia de propuestas concretas de perfeccionamiento al currículo actual. El tema fue abordado desde la necesidad de la inclusión de un grupo de materias relacionadas a la contabilidad internacional.

Algunos estudios revelaron dificultades con respecto a la carga horaria. Asimismo, manifestaron la necesidad de algunas asignaturas que ayudarían a completar la formación en temas de pertinencia internacional, o habilidades relacionadas a las nuevas tecnologías de la información y las comunicaciones. En ese sentido, algunas evidencias revelaron que muchas instituciones presentan elevadas cargas de asignaturas de contenido básico en detrimento de otras como Tecnologías de la Información, Contabilidad internacional, Comercio Internacional, Derecho Comercial y Contabilidad Avanzada (Cella, Miranda \& Katsumi, 2011; Melo, 2013). Generalmente, los estudios publicados tomaron como antecedentes las insuficiencias en el tratamiento del tema. Además, se constató que muchos profesores no están conscientes de la existencia de las IES (Jacomossi \& Biavatti, 2017; Ramos, Miranda \& Moura, 2017).

Los autores resaltan la preocupación por que los estudiantes de contabilidad no estén suficientemente preparados para lidiar con los desafíos de la profesión en la actualidad. Siguiendo esta línea, los principales desafíos que sobresalen se asocian a las habilidades y atributos comunicativos, computacionales, analíticos, intelectuales, multidisciplinares e interdisciplinarios, a los conocimientos de asuntos globales, y al desarrollo de un pensamiento crítico (Bona, Katsumi, Tozetti \& Regis, 2012; Ramos et al., 2017).

Aunque los frutos derivados de la producción académica y científica son escasos, los autores dejan importantes valoraciones sobre las deficiencias en la adaptación de los currículos a los estándares internacionales. En líneas generales, se reconoce que, aunque se percibe un estímulo de los docentes hacia la actualización y perfeccionamiento de la docencia, la gestión del proceso de enseñanza aún se centra en aspectos técnicos, en detrimento del desarrollo de habilidades interpersonales, éticas y comunicativas (Tonetto, Lima, Ferreira \& Gomes, 2013; Cunha, Guidini \& Klann, 2017; Alves, Kronbauer, Ott \& Thomaz, 2017).

El tratamiento de la temática en los cursos de posgrado en funcionamiento en Brasil se realizará a partir de la información que muestra la tabla 2 .

En la tabla 2, se puede observar que predomina la clasificación 'optativa' para la asignatura Metodología de la Enseñanza. Los contenidos impartidos centran su atención en los actores del proceso (profesor y legislaciones institucionales) y en los componentes del mismo (evaluación y método). Entonces, se observa la intención de profundizar en los atributos necesarios para los profesores, pero con limitado tratamiento de la epistemología de la educación contable.

A partir de la revisión del contenido de la asignatura Metodología de la Enseñanza, no se observa que se aborden los temas sobre las IES. El contenido desarrollado se basa en el intento por solventar las falencias del desempeño docente. Para que los contenidos subyacentes en las normas internacionales de contabilidad puedan ser integrados a las nuevas dinámicas de la educación y al escenario profesional contable, deben ser objeto de la enseñanza. Evidentemente, las nuevas realidades exigen a la profesión una adaptación y/o reorientación de sus planes de estudios, así como un espacio de discusión sobre aspectos propios y derivados del proceso de formación para que ayuden a agilizar y concretar reformas curriculares y académicas pertinentes y significativas. En este sentido, 
la idea es propiciar contenido que se oponga al tratamiento individual y aislado de los componentes didácticos o elementos que participan en el proceso de enseñanza-aprendizaje. En su lugar, habría que utilizar una concepción más integradora del contenido u objeto del proceso. Se requiere de rigurosidad teórica, metodológica y epistemológica para que los profesionales de contabilidad se posicionen como gestores de conocimiento que puedan ofrecer respuestas más aproximadas a la solución de las necesidades y problemas profesionales en el marco de un objetivo definido por la Normativa Internacional de Educación Contable.

Tabla 2. Informaciones sobre los cursos de posgrado revisados (período 2010-2017)

\begin{tabular}{|c|c|c|c|c|}
\hline Programa & Universidad perteneciente & $1^{*}$ & $2^{*}$ & $2^{* *}$ \\
\hline FURB & $\begin{array}{l}\text { Universidad de Blumenau } \\
\text { Santa Catarina }\end{array}$ & 5 & $\begin{array}{l}1 \text { (Opta- } \\
\text { tiva) }\end{array}$ & 3 \\
\hline UFSC & $\begin{array}{l}\text { Universidad Federal de } \\
\text { Santa Catarina }\end{array}$ & - & $\begin{array}{l}1 \text { (Opta- } \\
\text { tiva) }\end{array}$ & 2 \\
\hline USP & Universidad de Sáo Paulo & 3 & $\begin{array}{l}1 \text { (Opta- } \\
\text { tiva) }\end{array}$ & 1 \\
\hline UFU & $\begin{array}{l}\text { Universidad Federal de } \\
\text { Uberlândia }\end{array}$ & 3 & $\begin{array}{l}1 \text { (Opta- } \\
\text { tiva) }\end{array}$ & 1 \\
\hline UNB & Universidad de Brasilia & 2 & $\begin{array}{l}1 \text { (Opta- } \\
\text { tiva) }\end{array}$ & 2 \\
\hline UFRJ & $\begin{array}{l}\text { Universidad Federal de Rio } \\
\text { de Janeiro }\end{array}$ & 1 & $\begin{array}{l}1 \text { (Obli- } \\
\text { gatoria) }\end{array}$ & - \\
\hline UNIOESTE & $\begin{array}{l}\text { Universidad Estadual Oeste } \\
\text { de Paraná }\end{array}$ & 1 & $\begin{array}{l}1 \text { (Obli- } \\
\text { gatoria) }\end{array}$ & - \\
\hline UFPB/ JP & $\begin{array}{l}\text { Universidad Federal de } \\
\text { Paraíba }\end{array}$ & 1 & $\begin{array}{l}1 \text { (Obli- } \\
\text { gatoria) }\end{array}$ & - \\
\hline UFC & $\begin{array}{l}\text { Universidad Federal de } \\
\text { Ceará }\end{array}$ & 1 & - & 3 \\
\hline UNISINOS & $\begin{array}{l}\text { Universidad del Vale Río } \\
\text { de Sinos }\end{array}$ & - & $\begin{array}{c}1 \text { (Opta- } \\
\text { tiva) }\end{array}$ & 1 \\
\hline UEM & $\begin{array}{l}\text { Universidad Estadual de } \\
\text { Maringá }\end{array}$ & - & $\begin{array}{l}1 \text { (Opta- } \\
\text { tiva) }\end{array}$ & 1 \\
\hline FURG & $\begin{array}{l}\text { Universidad Federal Río } \\
\text { Grande }\end{array}$ & - & $\begin{array}{l}1 \text { (Opta- } \\
\text { tiva) }\end{array}$ & - \\
\hline UERJ & $\begin{array}{l}\text { Universidad estadual Río de } \\
\text { Janeiro }\end{array}$ & - & $\begin{array}{l}1 \text { (Opta- } \\
\text { tiva) }\end{array}$ & - \\
\hline
\end{tabular}

\begin{tabular}{|c|c|c|c|c|}
\hline Programa & Universidad perteneciente & $1^{*}$ & $2^{*}$ & $2^{* *}$ \\
\hline UFRGS & $\begin{array}{l}\text { Universidad Federal Río } \\
\text { Grande do Sul }\end{array}$ & - & $\begin{array}{l}1 \text { (Opta- } \\
\text { tiva) }\end{array}$ & - \\
\hline UFRN & $\begin{array}{l}\text { Universidad Federal Río } \\
\text { Grande do Norte }\end{array}$ & - & $\begin{array}{l}1 \text { (Opta- } \\
\text { tiva) }\end{array}$ & - \\
\hline FECAP & $\begin{array}{l}\text { Fundación Álvares Penteado } \\
\text { São Paulo }\end{array}$ & - & $\begin{array}{l}1 \text { (Opta- } \\
\text { tiva) }\end{array}$ & - \\
\hline USP-RP & $\begin{array}{l}\text { Universidad Campus } \\
\text { Riberão Preto São Paulo }\end{array}$ & - & $\begin{array}{l}1 \text { (Opta- } \\
\text { tiva) }\end{array}$ & - \\
\hline PUC/SP & $\begin{array}{l}\text { Pontifícia Universidad } \\
\text { Católica São Paulo }\end{array}$ & - & $\begin{array}{c}1 \text { (Opta- } \\
\text { tiva) }\end{array}$ & - \\
\hline $\begin{array}{l}\text { UNOCHA- } \\
\text { PECO }\end{array}$ & $\begin{array}{l}\text { Universidad de la Región } \\
\text { Chapecó Santa Catarina }\end{array}$ & - & $\begin{array}{l}1 \text { (Opta- } \\
\text { tiva) }\end{array}$ & - \\
\hline UFPE & $\begin{array}{l}\text { Universidad Federal de } \\
\text { Pernambuco }\end{array}$ & - & $\begin{array}{c}1 \text { (Opta- } \\
\text { tiva) }\end{array}$ & - \\
\hline UFMG & $\begin{array}{l}\text { Universidad Federal de } \\
\text { Minas Gerais }\end{array}$ & - & $\begin{array}{l}1 \text { (Opta- } \\
\text { tiva) }\end{array}$ & - \\
\hline UFPR & $\begin{array}{l}\text { Universidad Federal de } \\
\text { Paraná }\end{array}$ & - & $\begin{array}{c}1 \text { (Opta- } \\
\text { tiva) }\end{array}$ & - \\
\hline UFRPE & $\begin{array}{l}\text { Universidad Federal de } \\
\text { Pernambuco }\end{array}$ & - & $\begin{array}{l}1 \text { (Opta- } \\
\text { tiva) }\end{array}$ & - \\
\hline UFBA & $\begin{array}{l}\text { Universidad Federal de } \\
\text { Bahía }\end{array}$ & - & $\begin{array}{l}1 \text { (Opta- } \\
\text { tiva) }\end{array}$ & - \\
\hline UFMS & $\begin{array}{l}\text { Universidad Federal de } \\
\text { Mato Grosso }\end{array}$ & - & $\begin{array}{l}1 \text { (Opta- } \\
\text { tiva) }\end{array}$ & - \\
\hline UFES & $\begin{array}{l}\text { Universidad Federal de } \\
\text { Espírito Santo }\end{array}$ & - & $\begin{array}{l}1 \text { (Opta- } \\
\text { tiva) }\end{array}$ & - \\
\hline FIPECAFI & $\begin{array}{l}\text { Fundación de Investigacio- } \\
\text { nes Contables São Paulo }\end{array}$ & - & - & - \\
\hline FUCAPE & $\begin{array}{l}\text { Escuela de Negocios Espí- } \\
\text { rito Santo }\end{array}$ & - & - & - \\
\hline $\begin{array}{l}\text { FUCAPE- } \\
\text { MA }\end{array}$ & $\begin{array}{l}\text { Escuela de Negocios Minas } \\
\text { Gerais }\end{array}$ & - & - & - \\
\hline UFG & $\begin{array}{l}\text { Universidad Federal de } \\
\text { Goiás }\end{array}$ & - & - & - \\
\hline UPM & $\begin{array}{l}\text { Universidade Presbiteriana } \\
\text { Mackenzie São Paulo }\end{array}$ & - & - & - \\
\hline$\sum$ & & 17 & 25 & 14 \\
\hline
\end{tabular}

(1*) Espacio académico 1: artículos sobre el tema de autores pertenecientes a los programas (tabla 1)

$\left(2^{*}\right)$ Espacio académico 2: programas que ofertan la disciplina Metodología de Enseñanza de forma optativa u obligatoria

$\left(2^{* *}\right)$ Espacio académico 2: tesis y disertaciones defendidas que, en alguna medida, abordan el tema.

Fuente: Elaboración propia a partir de datos de la plataforma sucupira CAPES. 
Quizá, el contenido actual de la asignatura Metodología de la Enseñanza no es lo suficientemente atractivo. Por ello, desde la propia concepción de los gestores de los programas, se considera la asignatura como optativa, ya que se piensa que su contenido no afectaría en mayor medida la competitividad del estudiante. Sin embargo, de ser enriquecido con el arsenal epistemológico necesario, el contenido de la asignatura señalado sería necesario para todos los estudiantes de posgrado en Contabilidad, que es el espacio para interiorizar la actitud esperada del contador.

En cuanto a la producción académica derivada de las disertaciones y tesis defendidas, en la tabla 2 , se puede observar que se desarrollaron catorce disertaciones sobre el tema de investigación. En cuanto al contenido tratado, se considera que las ideas y consideraciones aún se encuentran en un estado incipiente, pues se plantean en el nivel de comparación, el de caracterización y el de diagnóstico (Erfurth, 2009; Frosi, 2013; Silva, 2014; Caetano, 2014). La disertación de Santos (2017) promete un sustento teórico en las IES, pero no fue posible advertir consideraciones sobre brechas actuales y posibilidades de perfeccionamientos con enfoques integrales. El trabajo de Bettin (2017) se muestra más avanzado en cuanto a algunas directrices y sugerencias para cambios, aunque no aborda una discusión teórica y práctica sobre propuestas de perfeccionamientos de currículos para la formación contable.

Para analizar los programas que han realizado acciones más integrales en cuanto al tema de las IES, se vincularon los artículos publicados con el programa de origen del autor principal o con el programa al cual tributa las investigaciones del autor principal. Puede observarse que, en los programas FURB, USP, UNB y UFU, las acciones en el tratamiento del tema han sido más integradas. Otros programas han realizado alguna acción en el abordaje del tema, como es el caso de los programas UFRJ, UNIOESTE, UFPB, UNISINOS y UEM. De esta forma, puede plantearse que, en los documentos revisados del 64\% de los programas en funcionamiento, no se ha observado de forma explícita acciones que evidencien el tratamiento del contenido recogido en las IES.

\section{Consideraciones finales}

Los criterios para considerar el corte humanista como la base angular de la labor educativa para la formación de los contadores han despertado el interés de un gran número de estudios; asimismo, ha habido consenso en algunos aspectos relacionados a dicho enfoque. Sin embargo, estos no se encuentran suficientemente representados en la Normativa Internacional de Educación Contable, por lo que han surgido más discusiones al respecto. En ese sentido, las IES propuestas por el IFAC no son suficientes para resolver los problemas formativos del profesional contable. El debate se ha desarrollado de manera amplia y profunda. De esta forma, se han puesto en evidencia los principales problemas de las IES, centrados en puntos neurálgicos del proceso de formación contable. El debate continúa inconcluso, pues las propuestas de perfeccionamiento son insuficientes y limitadas, $y$ todavía no se encuentra la manera para potenciar la pluralidad y fuerza argumental de la contabilidad.

El análisis crítico de los diferentes autores evidenció que la Normativa Internacional de Educación Contable enfrenta diversas problemáticas. La principal incertidumbre radica en la formación de competencias: la ambigüedad en el tratamiento conceptual de habilidades y competencias amenaza el direccionamiento del proceso formativo hacia el desarrollo de las competencias relacionadas con los procesos complejos, inteligentes y cognitivos. Asimismo, la recomendación 
de un contenido programático con énfasis en lo contable-financiero convierte el currículo en una estructura rígida y descontextualizada que gravita sobre la conexión de la contabilidad con los referentes teóricos del saber. Así, no se propicia el cierre de la fisura entre teoría y práctica, por lo que el desarrollo de actitudes críticas y reflexivas que se requieren formar en el contador están en inminente peligro. La omisión de contenidos relacionados a aspectos sociales y ambientales ponen en riesgo la legitimidad, consistencia y coherencia de la contabilidad como disciplina social; además, y pone en duda su pretensión analítica frente a sus implicaciones sociales, políticas, éticas y morales.

Se ha de concordar que la principal limitante de las IES está en la importancia dada a la perspectiva pragmática como cimiento de la profesión contable-financiera. Bajo esta perspectiva, no se considera necesario que el proceso formativo contemple las herramientas para consolidar criterios analíticos y críticos. Por lo tanto, se elude la interdisciplinariedad y se quebrantan los compromisos con la formación de competencias más complejas. La inexistencia de sinergias entre diferentes disciplinas no permitirá formar competencias para que la información resultante de la contabilidad sea multidimensional. De esta manera, la carencia de fundamentos epistemológicos no permite expresar las problemáticas del contexto social, por lo que la magnitud de lo económico supone que se pierda de vista la responsabilidad que la contabilidad asume frente al contexto social en el que actúa.

Con relación a la repercusión de la Normativa Internacional de Educación Contable en los espacios académicos brasileños, puede afirmarse que se observan carencias argumentativas en las discusiones y el tratamiento de los principales conceptos de la normativa en los artículos publicados. Así, es pertinente sostener que la preocupación por la educación con- table no se ha situado en el contexto histórico de la problematización en torno a la normativa vigente. Se manifiesta una escasa necesidad por confrontar y pensar críticamente los elementos que movilizan a la profesión contable actual.

En la medida en que se avanzó y se confrontaron los actuales contenidos del programa analítico de las asignaturas didáctico-pedagógicas del posgrado, se constata que el énfasis continúa situándose en los métodos del proceso docente; por ello, se excluyen generalmente las perspectivas epistemológicas-pedagógicas subyacentes en los conceptos para la formación, tanto de los profesores como de los profesionales contables en general. De esta manera, se ha legitimado un quehacer didáctico con limitaciones de rigor crítico, conceptual y contextual en un escenario de internacionalización de la educación contable. En ese sentido, la asignatura didáctico-pedagógica del posgrado puede reconocer otros caminos y posibilidades temáticas esenciales para el abordaje de la educación contable; y para la formación de un contador poseedor de un pensamiento crítico y analítico acerca de la realidad humana, social y empresarial.

Para que el discurso científico y los contenidos de la disciplina del posgrado encuentren puntos convergentes, es necesario discutir sobre la importante educación filosófica del contador como sustento de su formación ciudadana. El contenido de una asignatura de educación contable no debe insistir exclusivamente en un contenido que sobrevalore los componentes técnicoinstrumentales, y las habilidades, las destrezas y los procedimientos manejados por los profesores. Los elementos conceptuales y las teorías de argumentación deben ser temas acuciantes en las propuestas pedagógicas para la formación ética del contador. Eso exige la concurrencia de diferentes saberes que aporten al proceso una hibridación interdisciplinaria. 
La aplicación de estas nuevas recomendaciones en educación contable aún está en estado embrionario, por lo que se requiere de mayor análisis, reflexión y perfeccionamiento. Aun así, si se pusieran en práctica los cambios mencionados, incluso con sus limitantes, determinarían un viraje en la forma de abordar el contenido de la profesión contable. La inclusión de otras dimensiones debe ser fuente de estudios para la legitimidad del carácter de disciplina social de la Contabilidad.

Este estudio muestra una recopilación de las ideas de los diferentes académicos e investigadores, lo que resulta importante para exponer el avance del conocimiento en términos de educación contable. La consolidación de las diferentes ideas y enfoques de los autores en un documento único facilitará el estudio y definición de lagunas para la continuidad de investigaciones sobre la temática. La opinión de los autores de este trabajo sobre las limitaciones de las IES puede ser retomada para realizar su aplicación práctica. En ese sentido, se espera que este estudio sea superado por otros que evidencien propuestas concretas de perfeccionamiento de la estructura de contenidos para la asignatura didáctico pedagógica del posgrado en Ciencias Contables.

\section{Referencias}

Alves, D., Kronbauer, C., Ott, E. \& Thomaz, J. (2017). O ensino dos CPCs nos cursos de Ciências Contábeis em instituiçôes de ensino superior do Brasil. Revista Contemporânea de Contabilidade, 14(32), 48-70. https:// doi.org/10.5007/2175-8069.2017v14n32p48

Argueta, M. (2009). Ejes transversales en el currículo de la formación inicial de docentes. San José: Coordinación Educativa y Cultural Centroamericana, CECC/SICA.

Bettin, J. (2017). Percepção dos Resultados de Aprendizagem Referentes às Normas Internacionais de Educação: Um Estudo Sob a Ótica dos Acadêmicos de Contabilidade
(Dissertaçấo de Mestrado). Universidade Federal de Maringá, Departamento De Ciências Contábeis. Maringá, Brasil.

Bona, E., Katsumi, J., Tozetti, J. \& Régis, D. (2012). Um estudo sobre a pesquisa em educação contábil em periódicos internacionais: temáticas envolvendo as normas internacionais de contabilidade. ConTexto, 12(22), 59-73. Recuperado de https://seer.ufrgs.br/ConTexto/ article/view/30010 [Consulta: 11 de febrero de 2019].

Caetano, R. (2014). Ensino de Contabilidade Pública em IES da Região Norte do Brasil: Adequaçôes às Diretrizes Propostas pelo CFC (Dissertação de Mestrado). Universidade do Vale do Rio dos Sinos, Escola de Gestão e Negócios. São Leopoldo, Brasil.

Cardona, J. (2000). La educación contable: una nueva cultura. Contaduría Universidad de Antioquia, 38, 73-98. Recuperado de https://aprendeenlinea.udea.edu.co/ revistas/index.php/cont/article/view/25580/21125 [Consulta: 11 de febrero de 2019].

Cardona, J. \& Zapata, M. (2006). Educación contable: antecedentes, actualidad y prospectiva. Medellín: Universidad de Antioquia.

Carrillo, O. \& Jurado, P. (2017). La educación técnico profesional y las competencias para la ciudadanía. el caso de las comunas de la provincia de Concepción, Chile. Calidad en la Educación, 46, 133-164. https://doi. org/10.4067/S0718-45652017000100133

Cassiani, D. \& Zabaleta, M. (2016). Metodologías utilizadas en la enseñanza de contabilidad internacional: una propuesta. Revista Interamericana de Educación, Pedagogía y Estudios Culturales, 9(1), 107-123. Recuperado de http://revistariiep.com/wp-content/ uploads/2017/11/vol9-num1-06.pdf [Consulta: 15 de febrero de 2019].

Castillo, C., Córdoba, J. \& Villarreal, J. (2014). Estándares Internacionales de educación (IES) en contabilidad y aseguramiento: nuevos retos de la profesión contable. Tendencias, XV(2), 118-135. https://doi. org/10.22267/rtend.141502.45 
Celerino, S. (2015). Currículos dos cursos de contabilidade: discussão em uma perspectiva educacional moderna e pós-moderna. Enfoque Reflexão Contábil, 34(2), 15-36. https://doi.org/10.4025/enfoque.v34i2.26976

Cella, G., Miranda, J. \& Katsumi, J. (2011). Contabilidade internacional: análise dos periódicos internacionais sobre pesquisas em educação contábil face à convergência e globalização. Revista Contemporânea de Contabilidade, 8(15), 177-198. https://doi. org/10.5007/2175-8069.2011v8n15p177

Cortés, J. (2006). El pensamiento crítico: Algunas reflexiones en torno a la educación contable. En Universidad de Manizales (ed.), Cuarto Foro de Educación Contable (pp. 257-262). Manizales: Universidad de Manizales. Recuperado de http:/www.scielo.org.co/ scielo.php?script=sci_nlinks\&ref=000131\&pid=S19003803201400010001000004\&lng=en [Consulta: 15 de febrero de 2019].

Crawford, L., Helliar, C., Monk, E. \& Veneziani, M. (2014). International Accounting Education Standards Board: Organizational Legitimacy within the Field of Professional Accountancy Education. Accounting Forum, 38, 67-89. https://doi.org/10.1016/j.accfor.2013.09.001

Cuenú, J. (2010). Impedimentos de los estudiantes de Contaduría Pública para formarse como sujetos constructores de conocimiento científicos. Revista internacional Legis de Contabilidad y Auditoría, 44,119-137.

Cunha, L., Guidini, A. \& Klann, R. (2017). A disciplina de Teoria da Contabilidade e a convergência às Normas Internacionais de Contabilidade: percepção dos professores dos Programas de Pós-graduação stricto sensu em Contabilidade. Pensar Contábil, 19(68), 4-16. Recuperado de http://www.atena.org.br/revista/ojs-2.2.3-06/index.php/pensarcontabil/article/ view/3046 [Consulta: 13 de febrero de 2019].

Czesnat, A., Cunha, J. \& Domingues, M. (2009). Análise Comparativa entre os Currículos dos Cursos de Ciências Contábeis das Universidades do Estado de Santa Catarina Listadas no MEC com o Currículo
Internacional Proposto pela UNCTAD/ONU/ISAR. Gestão e Regionalidade, 25(75), 22-30. doi: 10.13037/ gr.vol25n75.186

Da Silva, J. (2012). Sistema Federal Brasileiro de Custos: uma análise comparativa à luz das recomendaçóes da IFAC. Gestão, Finanças e Contabilidade, 2(3), 39-55. https://doi.org/10.29386/rgfc.v2i3.67

Dellaportas, S., Leung, P., Cooper, B., Jackling, B. (2006). IES4-ethics education revisited. Australian Accounting Review, 16(38), 4-12. https://doi.org/10.1111/j.18352561.2006.tb00321.x

Erfurth, A. (2009). Currículo Mundial e o ensino de contabilidade: Estudo dos Cursos de Graduação em Ciências Contábeis em Instituiçôes de Ensino Superior Brasileiras e Argentinas (Dissertação de Mestrado) Universidade Federal de Blumenau, Faculdade de Contabilidade. Blumenau, Brasil.

Erfurth, A. \& Carvalho, M. (2013). Currículo Mundial e o Ensino de Contabilidade: Estudo dos Cursos de Graduação em Ciências Contábeis em Instituiçóes de Ensino Superior Brasileiras e Argentinas. Contexto, 13(23), 47-60. Recuperado de https://seer.ufrgs.br/ ConTexto/article/view/30444 [Consulta: 14 de febrero de 2019].

Federación Internacional de Contadores (IFAC). (2008). Manual de los pronunciamientos internacionales de formación. Nueva York: IFAC.

Frosi, M. (2013). Um Estudo Sobre o Alinhamento dos Curriculos dos Cursos de Ciências Contábeis de IES da Região do Sul do Brasil Com As Propostas do Currículo Mundial (Dissertação de Mestrado). Universidade do Vale do Rio dos Sinos, Escola de Gestão e Negócios, São Leopoldo, Brasil.

Galagovsky, L. \& Adúriz, A. (2001). Modelos y analogías en la enseńanza de las ciencias naturales. El concepto de modelo didáctico analógico. Enseñanza de las Ciencias, 9(2), 231-242. Recuperado de https://www.raco.cat/ index.php/ensenanza/article/viewFile/21735/21569 [Consulta: 14 de febrero de 2019].

Contabilidad y Negocios (15) 30, 2020 / ISSN 1992-1896 
García, M. (2014). Enseńanza de la contabilidad como disciplina académica: Concepciones de ciencias del profesorado y pensamiento crítico. Entramado, 10(1) 164-174. Recuperado de http://www.scielo.org.co/ scielo.php?pid=S1900-38032014000100010\&script=sci_ abstract\&tlng=es [Consulta: 14 de febrero de 2019].

Giraldo G. (2006). Problemáticas de la formación del estudiante de contaduría pública: la pertinencia de la actitud científica y epistemológica. En Universidad de Manizales (ed.), Cuarto Foro de Educación Contable (pp. 204-212). Manizales: Universidad de Manizales. Recuperado de www.redalyc.org/html/2654/265431574010/ [Consulta: 14 de febrero de 2019].

Gómez, M. (2006). Comentarios sobre el aprendizaje-construcción en la teoría contable. Lúmina, 7, 129-153. https://doi.org/10.30554/lumina.07.1180.2006

Gómez, M. (2007). Las insuficiencias disciplinares de los estándares internacionales de educación -IES- para contadores profesionales. Lúmina, 8(13), 24-42. https://doi.org/10.30554/lumina.08.1183.2007

Gómez M. (2009). Tensiones, posibilidades y riesgos de la contabilidad medioambiental empresarial. Contaduría Universidad de Antioquia, 54, 55-78. Recuperado de https://aprendeenlinea.udea.edu.co/revistas/index. php/cont/article/view/7963 [Consulta: 12 de febrero de 2019].

Gómez, M. (2011). Pensando los fundamentos de la contabilidad como disciplina académica. Lúmina 12(14), 120-151. https://doi.org/10.30554/ lumina.12.696.2011

Gómez, M. \& Ospina, C. (eds.) (2009). Avances interdisciplinarios para una comprensión critica de la contabilidad. Medellín: Universidad de Antioquia-Universidad Nacional de Colombia.

Gómez, Y. \& Martínez, A. (2014). La investigación en el currículo: un reto para los programas de contaduría pública en Colombia. En Universidad Nacional Autónoma de México, Asociación Nacional de Facultades y Escuelas de Contaduría y Administración, Asociación
Latinoamericana de Facultades y Escuelas de Contaduría y Administración (coords.), XIX Congreso Internacional de Contaduría, Administración e Informática [memoria]. México, D. F.: UNAM, ANFECA, ALAFEC.

González, R. (2008). ¿Cuál es la importancia de la capacidad de pensamiento crítico en la enseñanza contable? Adversia, 3, 1-9. Recuperado de http://aprendeenlinea. udea.edu.co/revistas/index.php/adversia/article/viewFile/1978/1624 [Consulta: 14 de febrero de 2019].

González, V. (2006). La formación de competencias profesionales en la universidad. Reflexiones y experiencias desde una perspectiva educativa. Revista de Educación, 8, 175-187. Recuperado de https://core.ac.uk/download/pdf/41563302.pdf [Consulta: 19 de febrero de 2019].

Gracea, M. (2014). Enseńanza de la Contabilidad como Disciplina Académica: Concepciones de Ciencias del Profesorado y Pensamiento crítico. Entramado, 10(1), 164-174. Recuperado de http://www.scielo.org.co/ scielo.php?pid=S1900-38032014000100010\&script=sci_ abstract\&tlng=es [Consulta: 19 de febrero de 2019].

Gracia, E. (2000). Situación actual de la investigación contable en Colombia [ponencia]. IX Foro Internacional: Nuevas Perspectivas del Conocimiento Contable. Bogotá. Recuperado de file://C:/Users/User/Downloads/4331386-1-PB.pdf [Consulta: 12 de febrero de 2019].

Helliar, C. (2013). The Global Challenge for Accounting Education. Accounting Education: an International Journal, 22(6). 510-521. https://doi.org/10.1080/09 639284.2013 .847319

Izquierdo, M. \& Adúriz, A. (2003). Epistemological foundations of school science. Science and Education, 12(1), 27-43. https://doi.org/10.1023/A:1022698205904

Jacomossi, F. \& Biavatti, V. (2017). Normas Internacionais de Educação Contábil Propostas Pelo. International Accounting Education Standards Board. Evidenciaçâo Contábil \& Finanças, 5(3), 57-78. https://doi.org/10.18405/recfin20170304 
Larraín, A., Freire, P., Moretti, R., Requena, M. \& Sabat, B. (2015). ¿La Universidad en Chile promueve las habilidades de argumentación escrita? Un estudio exploratorio comparativo de estudiantes de educación universitaria y educación técnica. Calidad en la Educación, 43, 201-228. https://doi.org/10.4067/ S0718-45652015000200007

Larrinaga, C. (1999). Perspectivas alternativas de investigación en contabilidad: una revisión. Revista de Contabilidad, 2, 103-131. Recuperado de https:// dialnet.unirioja.es/servlet/articulo? codigo $=247958$ [Consulta: 20 de febrero de 2019].

León, F. (2009). La educación contable en el contexto anglosajón: una mirada a los años de construcción de comunidad. Cuadernos de Contabilidad, 10(27), 219245. Recuperado de https://revistas.javeriana.edu.co/ index.php/cuacont/article/view/3208 [Consulta: 21 de febrero de 2019].

León, G. (2014). La contabilidad frente a la hegemonía de racionalidad calculante. Lúmina, 15, 72-104. https:// doi.org/10.30554/lumina.15.1072.2014

López, L. (2013). Estándares internacionales y educación contable. Apuntes del CENES, 32(55), 239-261. https://doi.org/10.19053/22565779.2075

López, M. \& Rodríguez, L. (2003). Un caso de interdisciplinariedad en teoría de la contabilidad: Lo cambios contables desde el enfoque cognitivo-lingüístico. Encuentros Multidisciplinares, 15(5), 62-78. Recuperado de http://hdl.handle.net/10486/680283 [Consulta: 20 de enero de 2019].

Major, M. (2017). O positivismo e a pesquisa 'alternativa' em Contabilidade. Revista Contabilidade \& Fianaças, 28(74), 173-178. https://doi.org/10.1590/1808$057 \times 201790190$

Martínez, G. (2002). El rediseño curricular contable: entre lo profesional y lo disciplinar. 5campus.org, Contabilidad Internacional. Recuperado de http://ciberconta. unizar.es/leccion../rediseno/rediseno.pdf [Consulta: 25 de febrero de 2019].
Martínez, G. (2007). La educación contable en el nuevo contexto de significación. Contaduría Universidad de Antioquí, 50, 43-76. Recuperado de https://aprendeenlinea.udea.edu.co/revistas/index.php/cont/ article/view/2121/1731 [Consulta: 23 de febrero de 2019].

Martínez, G. (2008). La educación contable: encrucijada de una formación monodisciplinaria en un entorno complejo e incierto. Popayán: Editorial Universidad del Cauca, serie Estudios Sociales.

Martínez, G. (2010). Los desafíos de la educación contable: encrucijada de una formación monodisciplinaria en un entorno complejo e incierto. Porik An, 8(11), 13-72. Recuperado de http://www.unicauca.edu.co/ porik_an/imagenes_3noanteriores/No.11porikan/ articulo1.pdf [Consulta: 13 de febrero de 2019].

Marulanda, E., Piedrahita, J. \& Quintero, O. (2011). Actitud del estudiante de contaduría pública frente a su formación profesional y vida laboral. Adversia, VIII(1), 10-25. Recuperado de https://aprendeenlinea.udea.edu.co/revistas/index.php/adversia/article/ view/10953 [Consulta: 20 de febrero de 2019].

Mejías, E., Montes, C. \& Montillas, O. (2008). Fundamentos teóricos del modelo contable común para las pymes de América Latina: una alternativa a la regulación contable internacional IASB. Estudios Gerenciales, 24(107), 59-86. https://doi.org/10.1016/ S0123-5923(08)70037-8

Melo, H. (2013). Características da Produção Veiculada na Revista de Educação e Pesquisa em Contabilidade no Período de 2007 a 2012. Revista de Educação e Pesquisa em Contabilidade, 7(4), 424-443. https://doi. org/10.17524/repec.v7i4.973

Miranda, G. (2010). Docência universitária: uma análise das disciplinas na área da formação pedagógica oferecidas pelos programas de pós-graduação stricto sensu em Ciências Contábeis. Revista de Educação e Pesquisa em Contabilidade, 4(2), 81-98. https://doi. org/10.17524/repec.v4i2.202

Contabilidad y Negocios (15) 30, 2020 / ISSN 1992-1896 
Montoya, J. \& Farias, G. (2013). Formación en competencias de futuros contadores públicos: Una experiencia docente. Revista de Ciencias Sociales (RCS), XIX(3), 485 - 499. Recuperado de https://www.redalyc.org/ html/280/28028572011/ [Consulta: 12 de febrero de 2019].

Muñoz, S., Ruiz, G. \& Sarmiento, H. (2015). Didácticas para la formación en investigación contable: una discusión crítica de las prácticas de enseñanza. Revista Facultad de Ciencias Económicas: Investigación y Reflexión, 23(1) 53-86. https://doi.org/10.18359/ rfce. 608

Needles, E. (2008). International Education Standards (IES): Issues of Implementation - A Report on the Third IAAER Globalization Roundtable. Accounting Education: An International Journal, 17(1), 69-79. https://doi.org/10.1080/09639280802095206

Nersessian, N. \& Oliva, J. (2007). Reseña de razonamiento basado en modelos y cambio conceptual. Eureka, 4(3), 563-570. https://doi.org/10.25267/Rev_Eureka_ensen_divulg_cienc.2007.v4.i3.14

Plataforma Sucupira CAPES (2017). Recuperado de https://sucupira.capes.gov.br/sucupira/ [Consulta: 5 de enero de 2019].

Quijano, O. (2002). Armonización de la educación contable: el proyecto escolar de los gremios económicos. Cuadernos de Administración, 28, 101-117. https:// doi.org/10.25100/cdea.v18i28.133

Quinche, F. (2007). Una evaluación crítica de la contabilidad ambiental empresarial. Revista Facultad de Ciencias Económicas, XVI(1), 197-216. Recuperado de https://www.redalyc.org/articulo.oa?id=90916114 [Consulta: 14 de febrero de 2019].

Quintero, A. \& Ramirez, A. (2016). Por caminos contra-hegemónicos hacia una contabilidad ecológica. Contaduria Universidad de Antioquia, 68, 287-312. Recuperado de http://10495/7364/1/QuinteroAura_2016_CaminosContraHegemonicos.pdf [Consulta: 22 de febrero de 2019].
Quirós, E. (2006, abril-junio). Competencias formativas y mercado profesional contable. Revista Internacional Legis de Contabilidad \& Auditoría, (26), 144-195. Recuperado de file://C:/Users/User/Downloads/ competencias $\% 20$ formativas $\% 20 \mathrm{y} \% 20$ mercado $\% 20$ profesional $\% 20$ contable $\% 20$ (2).pdf [Consulta: 22 de febrero de 2019].

Ramírez, D. (2017). Análisis y desarrollo de la educación contable: temáticas de su abordaje y principales problemáticas según el pensamiento estudiantil socializado por la Fenecop (2000-2016). Cuadernos de Contabilidad, 18(46). https://doi.org/10.11144/Javeriana.cc18-46.adec

Ramos, V., Miranda, G. \& Moura, J. (2017). ENADE e Proposta Curricular do CFC: Um Estudo em Cursos Brasileiros de Ciências Contábeis. Revista de Educaçâo e Pesquisa em Contabilidade, 11(3), 261-275. https:// doi.org/10.17524/repec.v11i3.1479

Reyes, N. \& Chaparro, F. (2013). Metodologías activas para la enseńanza de las Normas Internacionales de Información Financiera en un ambiente virtual de aprendizaje. Cuadernos de Contabilidad, 14(36), 1147-1182. Recuperado de https://dialnet.unirioja. es/servlet/articulo?codigo=5470845 [Consulta: $22 \mathrm{de}$ febrero de 2019].

Rocancio, A., Mira, G. \& Muñoz, N. (2016). Las competencias en la formación del profesional contable: una revisión de las posturas institucionales y educativas en Colombia. Revista Facultad de Ciencias Económicas, 25(2), 83-103. https://doi.org/10.18359/rfce.3070

Rojas, W. (2002, diciembre). La educación contable en Colombia 1960-2000: Al servicio de la fraternidad económica moderna. Cuadernos de Administración, (28), 16-47.

Rojas, W. (2007, enero-marzo). Contribución de las ciencias sociales y humanas a la formación del contador público. Revista Internacional Legis de Contabilidad y Auditoría, 29, 145-172. Recuperado de http://legal. legis.com.co/document/Index?obra=rcontador\&docu 
ment=rcontador_7680752a7dcc404ce0430a010151 404c [Consulta: 22 de enero de 2019].

Rojas, W. (2008). Congoja por una educación contable fútil. Contaduria Universidad de Antioquia, 52, 259-274.

Rojas, W. \& Giraldo, G. (2015). Humanidades y formación contable: una relación necesaria para otear una reorientación de la profesión contable. Cuadernos de Contabilidad, 16(40), 261-276. https://doi. org/10.11144/Javeriana.cc16-40.hfcr

Romero, H. (2014). Problemas de investigación en contabilidad y problemas de la investigación en contabilidad. Revista Clio América, 8(15), 75-82. https:// doi.org/10.21676/23897848.830

Rueda, G. (2007). La investigación contable: vínculos ontológicos y las posibilidades de la investigación interpretativa. Contaduría Universidad de Antioquia, 50, 119-132. Recuperado de http://aprendeenlinea.udea.edu.co/revistas/index.php/cont/article/ view/2124/1734 [Consulta: 29 de enero de 2019].

Rueda, G. (2010a). El papel de la contabilidad ante la realidad más allá de la convergencia. Cuadernos de Contabilidad, 11(28), 149-169. Recuperado de https://revistas.javeriana.edu.co/index.php/cuacont/ article/view/3202 [Consulta: 19 de febrero de 2019].

Rueda, G. (2010b). Neoliberalismo y convergencia contable. Orígenes, características y propuestas. Lúmina, 11(1), 264-278. https://doi.org/10.30554/ lumina.11.1221.2010

Rueda, G. (2012). Los aportes de la teoría de la acción comunicativa y sus conceptos a una contabilidad para el entendimiento y la integración de la sociedad. Universitas Humanistica, 74, 227-263. Recuperado de http://revistas.javeriana. edu.co/index.php/univhumanistica/article/ view/3649/3188 [Consulta: 5 de febrero de 2019].

Rueda, G. \& Pinzón, J. (2009). Retos y transformaciones en la investigación formativa contable. Una reflexión en la reciente experiencia en la Pontificia Universidad Javeriana. En Memorias I Encuentro Nacional de
Profesores de Contaduría Pública (pp. 547-563). Bogotá: Pontificia Universidad Javeriana.

Rueda, G., Pinzón, J. \& Patiño, R. (2013). Los currículos de los programas académicos de contaduría pública, tras la enseñanza de lo internacional y la globalización en la contabilidad: Necesidades de ajuste más allá de respuestas técnicas. Cuadernos de Contabilidad, 14(35), 639-667. Recuperado de https://dialnet.unirioja.es/servlet/articulo?codigo $=5470860$ [Consulta: 21 de febrero de 2019].

Sánchez, W. (2011). La docencia en el lugar equivocado. Lúmina, (12), 240-259. https://doi.org/10.30554/ lumina.12.693.2011

Santos, G. (2017). A Percepção Sobre a Contribuição da Prática Contábil Simulada para a Aprendizagem Significativa: Estudo em uma Instituição Pública Federal de Ensino Superior (Dissertação de Mestrado). Programa de Pós-graduação em Ciências Contábeis, Universidade Federal de Uberlândia. Uberlândia, Brasil.

Selter, J. (2001). La aplicación de una didáctica creativa en la enseñanza de contabilidad. Revista Fuentes, 3, 87-106. Recuperado de https://revistascientificas. us.es/index.php/fuentes/article/viewFile/2735/2284 [Consulta: 23 de febrero de 2019].

Silva, E. (2014). Governança no Setor Público Segundo a IFAC - Estudo nas Universidades Federais Brasileiras (Dissertação de Mestrado). Universidade Federal do Ceará, Faculdade de Economia, Administração, Atuária e Contabilidade em Contabilidade. Ceará, Brasil.

Skliar, C. (2002, agosto). Alteridades y pedagogías. Educação \& Sociedade, (79), 85-123. https://doi.org/10.1590/ S0101-73302002000300007

Sosa, E. (2015). Las transformaciones del pensamiento contable en el desarrollo del capitalismo financiero: financiarización de la contabilidad y valor razonable. Revista Pensamiento Actual, 15(25), 15-26. Recuperado de file://C:/Users/User/Downloads/Dialnet-LasTransformacionesDelPensamientoContable [Consulta: 21 de enero de 2019].

Contabilidad y Negocios (15) 30, 2020 / ISSN 1992-1896 
Sugahara, S. \& Boland, G. (2010). The Role of Cultural Factors in the Learning Style Preferences of Accounting Students: A Comparative Study between Japan and Australia. Accounting Education: An International Journal, 19(3), 235-255. https://doi. org/10.1080/09639280903208518

Sugahara, S., Urasaki, N., Wei, M. \& Boland, G. (2010). The Effect of Students' Ethics Learning Experiences to Develop Ethical Reasoning Abilities: A Comparative Study between Japanese and Chinese Students. International Journal of Accounting, Auditing and Performance Evaluation, 6 (1), 54-79. https://doi. org/10.1504/IJAAPE.2010.030479

Sugahara, S. \& Wilson, R. (2013). Discourse Surrounding the IES for Professional Accountants. Ac-counting Education: An International Journal, 22(3), 213-232. https://doi.org/10.1080/09639284.2013.785860

Tamayo, O. \& Orrego, M. (2005). Aportes de la naturaleza de la ciencia y del contenido pedagógico del conocimiento para el campo conceptual de la educación en ciencias. Educación y Pedagogía, 17(43), 13-25. Recuperado de https://core.ac.uk/download/ pdf/13301299.pdf [Consulta: 20 de enero de 2019].

Tonetto, G., Lima, C., Ferreira, E. \& Gomes, J. (2013). Uma análise crítica entre os currículos dos cursos de Ciências Contábeis nos países do Mercosul e o proposto pela ONU/UNCTAD/ISAR. Evidenciação Contábil \& Finanças, 1(1), 85-98. https://doi. org/10.18405/recfin20130106

Villarreal, J., Cordoba J. \& Castillo, C. (2016). De la educación contable internacional al desarrollo de competencias. Espacios, 37(33), 1-8. Recuperado de www.revistaespacios.com/a16v37n33/16373305. html [Consulta: 22 de febrero de 2019].

Watty, K., Sugahara, S., Abayadeera, N., Perera, L. \& Mckay, J. (2014). Towards a Global Model of Accounting Education. Accounting Research Journal, 27(3), 286-300. https://doi.org/10.1108/ARJ-08-20130054

Zapata, Y. \& Sarmiento, J. (2016). Elementos de un debate epistemológico por la nueva educación contable. Teuken Bidikay, 9, 55-73. Recuperado de http://revistas. elpoli.edu.co/index.php/teu/article/view/1009 [Consulta: 22 de febrero de 2019].

Fecha de recepción: 06 de diciembre de 2019 Fecha de aceptación: 20 de julio de 2020 Correspondencia: xesther68@gmail.com gilbertojm@ufu.br marli.silva@ufu.br 